Proceedings of the 37th Polish Seminar on Positron Annihilation, Lądek-Zdrój 2007

\title{
Current Problems and Unknowns in Positronium Dynamics
}

\author{
G. DuplÂtre
}

Chimie Nucléaire, Institut Pluridisciplinaire Henri Curien

CNRS/IN2P3/ULP

23 rue du Loess, BP 28, F-67037 Strasbourg Cedex 2, France

\begin{abstract}
A survey is made on positronium dynamics in liquids. From literature data, it is shown that applying conventional kinetics to positronium reactions leads to quite satisfactory results in a large variety of solvents, solutes, reaction types and temperatures. On the opposite, applying the theoretically well established concepts of positronium bubble state and time-dependent reaction rate coefficient would imply the use of unrealistic solute radii in the related kinetic equations, with possible exception of large solutes (micelles). Some solutes displaying unexpectedly high reaction rate constants are shown to be refractory to any of the above quantitative approaches. The particular case of reversible positronium bound-state formation is further examined, the most consistent results arising, again, from the conventional analysis of the data.
\end{abstract}

PACS numbers: 36.10.Dr

\section{Introduction}

In spite of decades of studies on positronium (Ps) through positron annihilation lifetime spectroscopy (PALS) experiments, several questions regarding its dynamics in liquids have remained unanswered. The importance of gaining understanding is twofold. (i) On fundamental grounds, Ps may be considered as a paragon for theoretical chemical studies since it can undergo most of the chemical reactions. In addition, as a radical having a triplet ( $o-\mathrm{Ps})$ and singlet $(p$-Ps) states, it may suffer spin conversion reactions. (ii) As a probe of matter, the potential applications of Ps are manifold. Clearly, deriving quantitative information on a medium requires a profound and detailed knowledge of the Ps dynamics.

Although Ps reactions have been long treated through conventional approaches, two of its properties make it an exceptional species. (i) Ps can build a bubble around itself when present in a liquid, due to the repulsive forces exerted on both the electron and the positron by the surrounding molecules. Several models exist, aiming at defining the radius of the bubble, with the surface tension of the liquid as the driving parameter [1-3]. As compared to the radius of free Ps $(0.053 \mathrm{~nm})$, the existence of the Ps bubble, with a radius of about $0.4 \mathrm{~nm}$ in most liquids, should strongly affect the Ps diffusion coefficient. (ii) The $o$-Ps lifetime is 
short (in liquids, in the range 1-4 ns) and kinetic theories predict the reaction rate coefficient to be time-dependent (e.g., [4]). The consequences of this dependence have not yet been fully valued [5], but it is clear that they might not be marginal.

Among the many problems remained unsolved regarding Ps chemistry, the present contribution will be focussed on the contradictions appearing when confronting experimental results taken from the literature with the two concepts of Ps bubble state (BS) and time-dependence (TD) of the reaction rate coefficient. In addition, the particular case of Ps bound-state formation reactions will be scrutinised and inconsistencies inherent to the various models applied to this class of reactions will be pinpointed.

\section{Conventional approach to Ps dynamics in solutions: free Ps vs. bubble radii}

The interactions of $o$-Ps in liquids, either of a chemical (e.g., oxidation by a solute) or physical (e.g., trapping into micelles) nature, result in a decrease in its lifetime. Conventionally, the reaction scheme may be written as follows:

$$
2 \gamma \stackrel{\lambda_{3}(0)}{\longleftarrow} o-\mathrm{PS}+\mathrm{S} \stackrel{k^{\prime} C}{\longrightarrow} \text { products } \stackrel{\lambda_{\mathrm{p}}}{\longrightarrow} 2 \gamma
$$

$\lambda_{3}(0)=1 / \tau_{3}(0)$ is the $o$-Ps decay rate constant in the absence of reactant $(\mathrm{S}), k^{\prime}$ is the reaction rate constant, $C$ is the concentration of the reactant, and $\lambda_{\mathrm{p}}$ - the decay rate constant of the product. The effect of this "quenching" reaction on the measured lifetime, $\tau_{3}(C)$, is thus quantitatively expressed as

$$
1 / \tau_{3}(C)=1 / \tau_{3}(0)+k^{\prime} C
$$

Where $\tau_{3}(C)$ and $\tau_{3}(0)$ are the $o$-Ps lifetimes at concentration $C$ of solute and in the neat solvent, respectively.

For decades, information on the medium was sought by comparing the experimental $k^{\prime}$ with the diffusion-controlled reaction rate constant as given through the Schmoluchowski equation

$$
k_{\mathrm{D}}=4 \pi R \Delta N_{\mathrm{Av}} / 1000
$$

where $R$ is the sum of the reaction radii of the reactants, $\Delta$ - the sum of their diffusion coefficients and $N_{\mathrm{Av}}$ is Avogadro's number. In addition, the StokesEinstein equation is used, to express the diffusion coefficients, as

$$
D=k_{\mathrm{B}} T /\left(6 \pi \eta R_{\mathrm{h}}\right),
$$

where $k_{\mathrm{B}}$ is Boltzmann's constant, $T$ - the absolute temperature, $\eta$ - the viscosity, and $R_{\mathrm{h}}$ - the hydrodynamic radius of the reactant. By assuming that the reaction and hydrodynamic radii of a reactant are the same (which may not be correct in some cases), Eqs. (3) and (4) can be combined, yielding the well-known equation $[6]$ :

$$
k_{\mathrm{D}}=2 N_{\mathrm{Av}} k_{\mathrm{B}} T\left(2+R_{\mathrm{Ps}} / R_{\mathrm{S}}+R_{\mathrm{S}} / R_{\mathrm{Ps}}\right) /(3000 \eta) .
$$

Here, $R_{\mathrm{Ps}}$ and $R_{\mathrm{S}}$ are the Ps and solute radii, respectively.

Equation (5), with $R_{\mathrm{Ps}}$ taken as the free Ps radius $(0.053 \mathrm{~nm})$, has become very popular because it delivers excellent results in a very large number of cases. 
This is illustrated in Table I, for a variety of solutes, solvents, reaction types and temperatures: there is excellent agreement between the experimental $k^{\prime}$ and $k_{\mathrm{D}}$ calculated from Eq. (5). Many similar results are easily found in the literature.

\section{TABLE I}

Comparison of the Ps reaction rate constants as experimentally derived from Eq. (2), $k^{\prime}[2,7-9]$, or calculated from Eq. $(5), k_{\mathrm{D}}\left(R_{\mathrm{Ps}}=0.053 \mathrm{~nm}\right)$ and $k_{\mathrm{b}}\left(R_{\mathrm{Ps}}=R_{\mathrm{b}}\right.$, bubble radius); units: $\mathrm{M}^{-1} \mathrm{~ns}^{-1}$. Reactions: ox = oxidation, $\mathrm{sc}=$ spin conversion, bs $=$ bound state formation. Solutes: $\Phi \mathrm{NO}_{2}=$ nitrobenzene; HTEMPO $=4$-hydroxy2,2,6,6-tetramethylpiperidine-1-oxyl.

\begin{tabular}{|c|c|c|c|c|c|c|c|c|}
\hline Solute & Solvent & Reaction & $T[\mathrm{~K}]$ & $R_{\mathrm{S}}[\mathrm{nm}]$ & $R_{\mathrm{b}}[\mathrm{nm}]$ & $k^{\prime}$ & $k_{\mathrm{D}}$ & $k_{\mathrm{b}}$ \\
\hline $\mathrm{UO}_{2}^{2+}$ & water & ox & 294 & 0.212 & 0.291 & 9.7 & 10.4 & 6.8 \\
\hline \multirow[t]{4}{*}{$\mathrm{CrO}_{4}^{2-}$} & water & ox & 274 & 0.316 & 0.287 & 7.6 & 7.2 & 3.5 \\
\hline & & & 288 & & & 12.4 & 11.4 & \\
\hline & & & 298 & & 0.291 & 15.7 & 15.1 & 7.4 \\
\hline & & & 308 & & & 19.9 & 19.3 & \\
\hline \multirow[t]{3}{*}{$\Phi \mathrm{NO}_{2}$} & toluene & bs & 197 & 0.50 & & 4.3 & 4 & \\
\hline & & & 246 & & & 15.4 & 13.7 & \\
\hline & & & 278 & & & 25.6 & 24.7 & \\
\hline \multirow[t]{3}{*}{ HTEMPO } & benzene & $\mathrm{sc}$ & 294 & 0.35 & 0.408 & 22.7 & 21.9 & 10.1 \\
\hline & & & 323 & & & 38 & 41 & \\
\hline & m-cresol & ox & 294 & & 0.383 & 0.88 & 0.70 & 0.3 \\
\hline
\end{tabular}

Introducing the bubble radius, $R_{\mathrm{b}}$, as the Ps radius in Eq. (5) leads to an important decrease in the calculated reaction rate constant, $k_{\mathrm{b}}$, because of the resulting strong decrease in the Ps diffusion coefficient, see Eq. (4). This is illustrated in Table I, where $k_{\mathrm{b}}$ appears to be roughly half of $k^{\prime}$ whatever the solute, solvent and reaction type. In the table, $R_{\mathrm{b}}$ has been calculated by using one of the most popular models available [2]; using other models does not bring in any significant changes, the bubble radius being always calculated at about $0.4 \mathrm{~nm}$ independently of the solvent and temperature (lower value are obtained in water, due to its higher surface tension). More generally, a survey of some 20 data from the literature yielding $\mathrm{k}^{\prime}$ values in the range $0.5-40 \mathrm{M}^{-1} \mathrm{~ns}^{-1}$ shows a ratio $k_{\mathrm{D}} / k^{\prime}=0.99 \pm 0.06$, which is remarkably good (taking account also of the uncertainty on the solute radii) whereas $k_{\mathrm{b}} / k^{\prime}=0.45 \pm 0.06$.

\section{Effect of TD on the reaction rate coefficient}

Theory predicts that, on a short time scale (typically, below $1 \mu \mathrm{s}$ ), the reaction rate coefficient for a purely diffusion-controlled reaction should be time dependent according to [4]:

$$
k(t)=k_{\mathrm{D}}\left[1+R /(\pi \Delta t)^{1 / 2}\right],
$$

where $k_{\mathrm{D}}, R$, and $\Delta$ have been defined previously, and $t$ is the time. 
A major consequence of TD is that the decay profile of $o$-Ps is no more a simple decreasing exponential, the mathematical solution to the problem depending on whether the product of the reaction, $\tau_{\mathrm{p}}$ (see eq.1), has a lifetime longer or shorter than $\tau_{3}(0)$. For instance, in the case of Ps trapping in micelles, the product of the reaction is the trapped $o$-Ps, which possesses a longer lifetime than it has in water; the corresponding mathematical expression for the $o$-Ps decay, $F(t)$, reads as follows [5]:

$$
F(t)=A \exp \left(-\lambda_{\mathrm{p}} t\right)+B \exp \left(-\alpha t-\beta t^{1 / 2}\right)+C \exp \left(-\lambda_{\mathrm{p}} t\right) \operatorname{erf}(\delta t+\varepsilon) .
$$

$A, B, C, a, b, \delta$, and $\varepsilon$ are constants depending on $\tau_{3}(0), \tau_{\mathrm{p}}$ and $k_{\mathrm{D}}$ and $C$.

Assessing the effect of TD for the solutes listed in Table I cannot be made from experimental data as the PALS spectra are no more available. Therefore, the spectra were calculated from published PALS parameters, and the long-lived part (o-Ps decay) was fitted to the relevant $o$-Ps decay equation, including $k(t)$. A comparison of the various possible combinations between $k_{\mathrm{D}}$ or $k(t)$ and use of $R_{\mathrm{Ps}}$ or $R_{\mathrm{b}}$ as the Ps radius is shown in Table II, in the form of the solute radius, $R_{\mathrm{S}}$, extracted from the best fit to the data. Let us note that for the combination $k_{\mathrm{D}} / R_{\mathrm{Ps}}, R_{\mathrm{S}}$ is the same as in Table I and coincides with the effective size of the solute.

TABLE II

Comparison of the solute radius, $R_{\mathrm{S}}$, extracted from fitting the data according to various combinations: $C 1=k_{\mathrm{D}} / R_{\mathrm{Ps}}, C 2=k_{\mathrm{D}} / R_{\mathrm{b}}, C 3=k(t) / R_{\mathrm{Ps}}, C 4=$ $k(t) / R_{\mathrm{b}} ; \eta$ is the viscosity. Symbols as in Table I.

\begin{tabular}{|c|c|c|c|c|c|c|c|}
\hline \multirow[t]{2}{*}{ Solute } & \multirow[t]{2}{*}{ Solvent } & \multirow[t]{2}{*}{ Reaction } & \multirow[t]{2}{*}{$\eta[\mathrm{cp}]$} & \multicolumn{4}{|c|}{$R_{\mathrm{S}}[\mathrm{nm}]$ according to combination \# } \\
\hline & & & & $C 1$ & $C 2$ & $C 3$ & $C 4$ \\
\hline $\mathrm{UO}_{2}^{2+}$ & water & ox & 0.98 & 0.212 & 1.16 & 0.29 & 0.52 \\
\hline \multirow[t]{2}{*}{$\mathrm{CrO}_{4}^{2-}$} & water & ox & 1.70 & 0.316 & 1.74 & 0.44 & 0.62 \\
\hline & & & 0.72 & & & 0.45 & 0.86 \\
\hline \multirow[t]{2}{*}{$\Phi \mathrm{NO}_{2}$} & toluene & bs & 1.10 & 0.5 & 2.76 & 0.65 & 0.81 \\
\hline & & & 0.72 & & & 0.64 & 0.91 \\
\hline \multirow[t]{2}{*}{ HTEMPO } & benzene & $\mathrm{sc}$ & 0.65 & 0.35 & 1.92 & 0.56 & 0.74 \\
\hline & m-cresol & ox & 20.5 & 0.35 & & 0.43 & 0.27 \\
\hline
\end{tabular}

In the absence of TD, showing $R_{\mathrm{S}}$ instead of $k_{\mathrm{D}}$ (Table I) sets off the strong effect of using the bubble radius (C2) instead of the free Ps radius $(C 1): R_{\mathrm{S}}$ is about 5.5 times larger than the known solute radius. With TD, using $R_{\mathrm{Ps}}$ in the fitting results in a $R_{\mathrm{S}}$ value which is higher but still reasonably close to the known value; using $R_{\mathrm{b}}$ gives better results than without TD, but still higher than with $R_{\text {Ps }}$. Two additional peculiarities appear on the hypothesis of TD: (i) the value of $R_{\mathrm{S}}$ may change with temperature; (ii) in m-cresol, $C 4$ gives a smaller $R_{\mathrm{S}}$ when using $R_{\mathrm{b}}$, this being due to the strong influence of viscosity in the last part of Eq. (6). 
From Tables I or II, it thus appears that, in strong contradiction with theoretical expectations, the experimental results are much better explained in the absence of both TD and BS.

\section{Effect of TD on the Ps yield and the process of Ps inhibition}

The complete PALS spectra (i.e., including the $p$-Ps and free positron components besides $o$-Ps) calculated on the basis of any of the combinations quoted in Table II are virtually indistinguishable. However, when considering the sole contribution from o-Ps decay, some differences appears. At long times, all decay profiles remain hardly distinguishable. However, as shown in Fig. 1, the decay profile calculated from $C 4$ (time dependence and bubble radius) shows a very marked hump at short times as compared to the other combinations. As a consequence, the intensity of the long-lived component is higher with $C 4$, theoretically the most likely, than with the other combinations. Since in the absence of solute the PALS spectra are the same whatever the combination, important changes are to be expected when deriving inhibition constants.

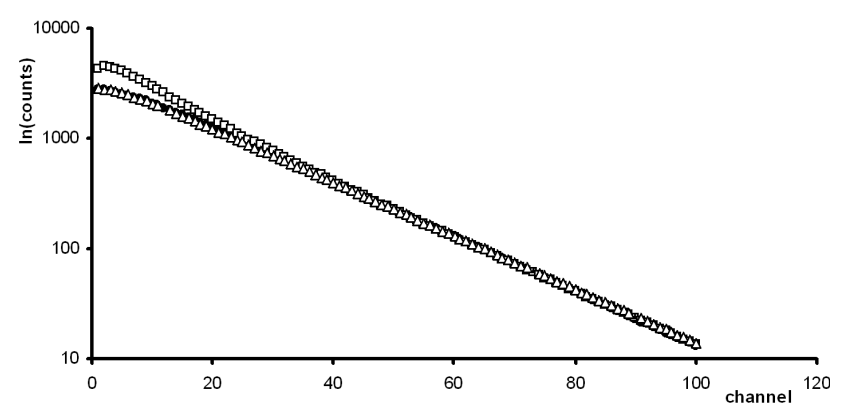

Fig. 1. Comparison of the long-lived component contribution in PALS spectra. Lower curves (indinstinguishable) obtained from $C 1(\bullet)$ and $C 3(\triangle)$; upper curve from $C 4(\square)$. All profiles merge into a single one roughly above $1.6 \mathrm{~ns}$. Basic spectrum $(C 1)$ calculated for $\mathrm{K}_{2} \mathrm{CrO}_{4}$ in water at $298 \mathrm{~K}: C=0.01 \mathrm{M}, k^{\prime}=15.7 \mathrm{M}^{-1} \mathrm{~ns}^{-1}, k_{\text {inhib }}=15 \mathrm{M}^{-1}$. Definition: $40 \mathrm{ps} /$ channel.

As an example, let us consider $\mathrm{CrO}_{4}^{2-}$, an oxidizer that promotes both $\mathrm{Ps}$ inhibition and quenching in water. A spectrum was calculated for $0.01 \mathrm{M}$, using $C 1$, from published data at $298 \mathrm{~K}[8,10]: k^{\prime}=15.7 \mathrm{M}^{-1} \mathrm{~ns}^{-1}, k_{\text {inhib }}=15 \mathrm{M}^{-1}$. The initial o-Ps intensity, $I_{3}(C)$, was supposed to obey the well documented expression [10]:

$$
I_{3}(C)=I_{3}(0) /\left(1+k_{\text {inhib }} C\right) .
$$

The $o$-Ps intensity in water at $298 \mathrm{~K}$ is $I_{3}(0)=28.5 \%$ [8]; the initial intensities of the longest-lived component in the calculated spectra were $24.8,26.0$, and $32.6 \%$ for $C 1, C 3$, and $C 4$, respectively. As noted above, $I_{3}$ appears to be highest with $C 4$. The consequences for $k_{\text {inhib }}$ are drastic, with $k_{\text {inhib }}=15,9.7$, and $-12.6 \mathrm{M}^{-1}$, respectively. Clearly, a negative value for $k_{\text {inhib }}$ makes no sense. 
The results just described come from fitting a PALS spectrum calculated with $C 1$; they might be somewhat different if an experimental PALS spectrum was to be analysed according to $C 4$. Nevertheless, the fact that, implicitly, the long-lived component should have a much higher intensity than if using any of the other combinations should result in very important changes in the inhibition constants derived.

\section{Solutes (apparently) reacting at faster rates than diffusion-controlled}

Tables I and II show some of the many solutes that obey the conventional approach (i.e., no TD and no BS) nicely. However, literature provides examples of solutes that appear to react more efficiently than expected from Eq. (5). This is the case, for instance, for iodine in various alkanes at $293 \mathrm{~K}[6]$. In the series of solvents pentane, hexane, octane, decane, the experimental $k^{\prime}$ values are in the order $115,85,40$, and $27 \mathrm{M}^{-1} \mathrm{~ns}^{-1}$ and are well correlated with the sequence of viscosities: $0.227,0.318,0.524,0.852 \mathrm{cp}$. However, using Eq. (5) with the known $I_{2}$ radius of $0.266 \mathrm{~nm}$ leads to ratios $\rho=k^{\prime} / k_{\mathrm{D}}$ that are $2.3,2.3,1.8$, and 2.0. Although this anomaly has not found explanation, a first thought might be that Ps would probe each individual iodine atoms in $I_{2}$, which would be equivalent to doubling the nominal concentration (i.e., the product $k^{\prime} C$ in Eq. 2). This observation is strongly reminiscent of what has been observed with $\mathrm{Fe}(\mathrm{CN})_{6}^{4-}$ ions whose inhibition constant, through positron capture, was found to be about 6 times that of the $\mathrm{CN}^{-}$ion [11]. However, this simple explanation seems insufficient when considering the same solute, $I_{2}$, in alcohols at $293 \mathrm{~K}$ [6]. For the sequence methanol, ethanol and butanol, one has $k^{\prime}=37,20,16 \mathrm{M}^{-1} \mathrm{~ns}^{-1}, \eta=0.584,1.21$, $3.04 \mathrm{cp}$ and $\rho=1.9,2.0,4.3$. Whereas $k^{\prime}$ is again correlated with $\eta$ on changing from methanol to ethanol, this is no more true for butanol, in which $\rho$ increases twofold.

Iodine might be suspected not to be an ideal solute to examine, as it is liable to hydrolysis in protic solvents. Let us note, however, that for diffusion-controlled reactions the exact nature of the solute is unimportant — only its radius plays a role. Nevertheless, other solutes may be found in the literature, presenting similar anomalies. Two of them are briefly examined in the following, with the aim of gaining a hint to the problem.

The first example is quinone. At $294 \mathrm{~K}$, for the sequence of solvents acetone, methanol, dimethylsulfoxide (DMSO), and octanol, one has $k^{\prime}=43,30,14$, and $7.5 \mathrm{M}^{-1} \mathrm{~ns}^{-1}$ for $\eta$ increasing as $0.584,1.21,2.17$, and $8.69 \mathrm{cp}$ [12-14]; with a radius estimated at $0.35 \mathrm{~nm}$, Eq. (5) results in $\rho=0.98,1.2,2.1$, and 4.5 . The values of $\rho$ for acetone and methanol suggest that $0.35 \mathrm{~nm}$ is a reasonable value for the quinone radius. Let us consider next the twofold increase observed in DMSO. The experimental information comes from the product $k^{\prime} C$ (see Eq. 2), so that any apparent increase in the reaction rate must come from an increase in either $k^{\prime}$ (or $k_{\mathrm{D}}$ ) or $C$. Taking account of the hint given by the behaviour of 
$I_{2}$, a reaction satisfying this requirement is dimerisation. In this case, considering literally the definition of $k_{\mathrm{D}}$ (somewhat proportional to $R_{\mathrm{S}}$ ) and $C$ (number of reacting species per unit volume), it is indeed expected that $k_{\mathrm{D}}$ would roughly increase (once dimerised, the molecules show a higher radius) whereas $C$ would not change (number of molecules per unit volume kept constant). On this hypothesis, higher aggregation processes (like tetramerisation) would yield higher values of $\rho$, as observed in octanol.

The second solute is the HTEMPO free radical (and, for octanol solutions only, the very similar TMOPO free radical: HTEMPO $=4$ - hydroxy-TMOPO). Table III shows a selection of $\rho$ values calculated from literature data $[2,14]$. The solute radius was taken as $0.35 \mathrm{~nm}$.

TABLE III

Comparison of experimental $k^{\prime}$ values and theoretical ratios, $\rho=k^{\prime} / k_{\mathrm{D}}$, for HTEMPO in various solvents (TMOPO, in octanol). NDMA $=N$, $N$-dimethylacetamide; NMFA $=N$-methylformamide. Other symbols as in Tables I and II.

\begin{tabular}{c|c|c|c|c}
\hline \hline Solvent & $T[\mathrm{~K}]$ & $\eta[\mathrm{cp}]$ & $k^{\prime}\left[\mathrm{M}^{-1} \mathrm{~ns}^{-1}\right]$ & $\rho$ \\
\hline benzene & 294 & 0.652 & 23 & 1.0 \\
methanol & 203 & 4.02 & 2.7 & 1.1 \\
& 223 & 2.29 & 5 & 1.06 \\
& 243 & 1.43 & 8.5 & 1.0 \\
NDMA & 323 & 0.693 & 23 & 1.0 \\
m-cresol & 294 & 16.9 & 0.88 & 1.1 \\
NMFA & 294 & 1.82 & 6.3 & 3.8 \\
octanol & 256 & 44 & 2.5 & 8.6 \\
& 294 & 8.7 & 6.3 & 3.8
\end{tabular}

The six first lines show values of $\rho$ that are very close to 1 , pointing to a correct estimation of the HTEMPO radius. As compared to these lines, the value of about 4 found for $\rho$ in NMFA and octanol at $294 \mathrm{~K}$ indicates that a hint to such abnormal ratios is not likely to be found by considering the viscosities. On the hypothesis of molecular association, HTEMPO and TMOPO appear to be prone to a rather high degree of aggregation in NMFA and octanol, respectively. Furthermore, the data suggest that, if true, this process could be studied in detail as a function of temperature, e.g.: $\rho$ might tend to 1 upon heating in octanol and some information might come on the way the molecules geometrically aggregate (in a dimer, for instance, the size of the aggregate is greatly different whether the constituents are linked through their extremities or are piled up).

It should be noted at this point that molecular association should not be considered as the only possible explanation to the anomalies. In particular, the fact 
that, for quinone and TMOPO in octanol, $k^{\prime}$ increases with an activation energy significantly lower than that of viscosity [14] might point to other directions.

From the preceding sections, the effect of BS alone is easily deduced: the $\rho$ values just commented are roughly increased twofold. Taking account of both BS and TD, as theory would recommend, does not lead to suppress the anomalous behaviours just depicted. This is illustrated in Table IV for quinone and TMOPO in octanol, in the form of the $R_{\mathrm{S}}$ values deduced from fitting the data according to combinations $C 1$ and $C 4$.

\section{TABLE IV}

Comparison of the solute radii, $R_{\mathrm{S}}$, and the inhibition constants, $k_{\text {inhib }}$, when fitting the data by using $C 1$ (left) or $C 4$ (right). Solvent: octanol. Expected radii: about $0.35 \mathrm{~nm}$.

\begin{tabular}{c|c|c|c|c|c}
\hline \hline Solute & $T[\mathrm{~K}]$ & \multicolumn{2}{|c|}{$R_{\mathrm{S}}[\mathrm{nm}]$} & \multicolumn{2}{c}{$k_{\text {inhib }}\left[\mathrm{M}^{-1}\right]$} \\
\hline quinone & 256 & 35 & 8.6 & 11 & 4.7 \\
& 294 & 20 & 10.5 & 7.5 & $<0$ \\
TMOPO & 256 & 41 & 8.3 & 2.5 & $<0$ \\
& 294 & 17 & 8.1 & 1.8 & $<0$
\end{tabular}

It may be seen that $C 1$ results in $R_{\mathrm{S}}$ values that are very large as compared to expectation $(0.35 \mathrm{~nm})$. The radii are smaller with $C 4$, but still much higher than expected; furthermore, $k_{\text {inhib }}$ as derived from the PALS intensities might even assume negative values.

\section{The case of large solutes: micelles in water}

In the last years, the Strasbourg group has carried experiments with the aim of demonstrating the usefulness of PALS to characterise quantitatively micellar systems. The first system chosen was such that the micelles had been well characterised previously, through conventional techniques (such as light or neutron scattering), in order to establish a general method in PALS. Thus, for sodium dodecylsulphate (SDS), the 4 combinations, $C 1$ through $C 4$, were used when fitting the spectra [5]. The results are shown in Table V.

It may be seen that only combinations $C 1$ (no BS, no TD) and $C 4$ (both BS and TD) lead to satisfactory results, with $R_{\text {mic }}$ values close to the known value. Thereafter, several experiments were carried out showing the aptitude of PALS to deliver qualitative (e.g., micellar shape) and quantitative (e.g., micellar radius, aggregation number) information. Taking account of the theoretical prescriptions, the PALS spectra were systematically analysed through $C 4$, giving good agreement whenever data from conventional techniques were also available (e.g., [15]). 


\section{TABLE V}

Comparison of the micellar core radius for SDS solutions in water at $294 \mathrm{~K}$ under various hypotheses. From literature data, the expected radius is $R_{\text {mic }}=1.7-1.8 \mathrm{~nm}$.

\begin{tabular}{c|c|c}
\hline \hline & $R_{\text {Ps }}=0.053 \mathrm{~nm}$ & $R_{\mathrm{b}}=3.11 \mathrm{~nm}$ \\
\hline$k_{\mathrm{D}}$ & 1.9 & 1.1 \\
$k(t)$ & 0.077 & 1.7
\end{tabular}

However, the possibility may exist of deriving similarly satisfactory results on the basis of the $C 1$ assumption.

\section{A particular case of Ps reactions: bound-state formation}

Nitrocompounds have soon been recognised to promote the specific reaction of bound-state formation with Ps [9]. A particular feature of this reaction lies in its reversibility, as revealed by the fact that $k^{\prime}$, derived from the data by using Eq. (2), shows a maximum when studied as a function of temperature $(T)$; the first reaction scheme proposed was thus as follows [9]:

$$
2 \gamma \stackrel{\lambda_{3}(0)}{\longleftarrow} o-\mathrm{Ps}+\mathrm{S} \stackrel{\stackrel{k_{1}}{\longrightarrow}}{\longleftarrow}[o-\mathrm{PsS}] \stackrel{\lambda_{\mathrm{c}}}{\longrightarrow} 2 \gamma .
$$

Here, $k_{1}$ and $k_{2}$ are the forward and backward reaction rate constants, respectively, and $\lambda_{\mathrm{c}}$ is the bound-state decay rate constant. It is easily shown that the overall reaction rate constant, $k^{\prime}$, as derived from Eq. (2), is a simple function of the various constants in scheme (9) [16].

A typical example is shown in Fig. 2, for nitrobenzene in toluene [9]. In a conventional approach, $k_{1}$ should obey Eq. (5), and $k_{2}$, the Arrhenius equation

$$
\begin{aligned}
& k_{1}=A T \exp \left(-E_{1} /\left(k_{\mathrm{B}} T\right)\right), \\
& k_{2}=B \exp \left(-E_{2} /\left(k_{\mathrm{B}} T\right)\right) .
\end{aligned}
$$

By assuming a bound-state lifetime equal to that of free $\mathrm{e}^{+}(0.425 \mathrm{~ns})$, fitting the data through Eqs. (9)-(11) gives an excellent fit to the data (see Fig. 2), with the following parameters:

- $A=1.43 \mathrm{M}^{-1} \mathrm{~ns}^{-1} \mathrm{~K}^{-1}$ which implies, from Eq. (5), $R_{\mathrm{S}}=0.47 \mathrm{~nm}$, which is quite likely for the nitrobenzene radius (see Table II).

- $E_{1}=0.068 \mathrm{eV}$, which compares well with the viscosity activation energy in toluene, $E_{\eta}=0.089 \mathrm{eV}$, particularly when noting that an Arrhenius dependence for $\eta$ may not hold over the whole $T$ range studied. Let us note that both $A$ and $E_{1}$ are essentially meant to fit the low $T$ region of the data (right hand in Fig. 2), when the reverse reaction is not yet effective.

$-B=6.41 \times 10^{18} \mathrm{~s}^{-1}$ and $E_{2}=0.635 \mathrm{eV}$.

The value of $B$ is very high: high frequency factors for decomposition of organic compounds are in the range $10^{12}-10^{13} \mathrm{~s}^{-1}$, with some rare quotations as 


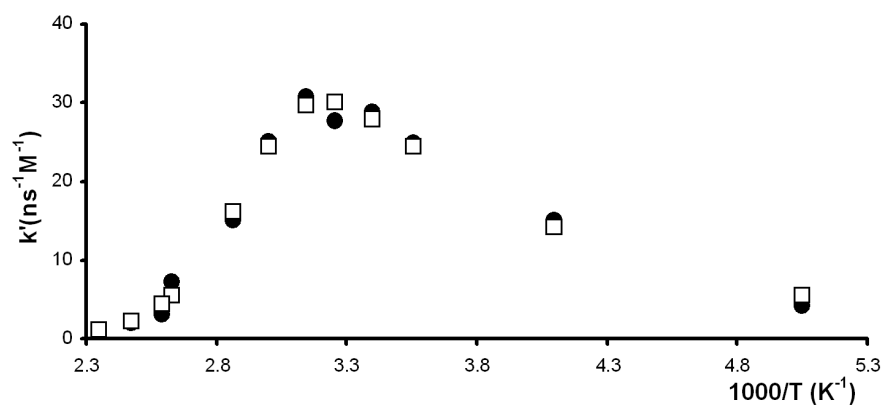

Fig. 2. Variation of the experimental reaction rate constant, $k^{\prime}$, as derived from Eq. (2), with $1 / T$. Experimental data $(\bullet)$ for nitrobenzene in toluene selected from [9]. Calculated values $(\square)$ from a fit by using Eqs. (9)-(11) with $R_{\mathrm{Ps}}=0.053 \mathrm{~nm}$.

high as $10^{17} \mathrm{~s}^{-1}$. But, on the other hand, $0.635 \mathrm{eV}$ is a high activation energy for the chemical decomposition of the bound-state, and the exponential in Eq. (10) can be balanced only by a high $B$ value. Furthermore, when considering the harmonic oscillator, the frequency is proportional to $m_{\mathrm{r}}^{-1 / 2}, m_{\mathrm{r}}$ being the reduced mass: by comparison with usual organic compounds, the reduced mass of a pair that would include Ps as in the Ps bound-states with nitrocompounds, should be much lower, resulting in a higher frequency. All in all, the high values of $A$ and $E_{2}$, which are necessary to account for the steepness of the decrease in $k^{\prime}$ after the turn-over $T$ is reached, seem to be at the limit of plausibility. Further efforts should be made both experimentally (e.g., analysing a larger set of data) and theoretically (e.g., estimation of the frequency factors for Ps bound-states) to unravel these anomalies.

Positronium bound-states have been the subject of several papers with the aim of determining the leading causes for their instability (e.g., [17-19]). All papers make use of the BS concept, and refer to the possibility of having or not a "bubble shrinking" upon bound-state formation, but almost none of them attempts any quantitative approach to the variation of $k^{\prime}$ with $T$. One exception comes from a series of papers by the Calcutta group [19]. As an explanation to the presence of the turn-over in the $k^{\prime}$ vs. $1 / T$ variation, the authors propose a two-step reaction in which the bound-state formation would depend on whether the Ps bubble can shrink when the two reactants come into contact. With reference to kinetic data from chemical systems showing an anti-Arrhenius dependence of the reaction rate constant, the authors explain the maximum as follows:

(i) At low temperature, diffusion is the dominant term in the expression for $k^{\prime}$ which can be written as in Eq. (9). The authors underline that the slope of the $\ln \left(k^{\prime}\right)$ vs. $1 / T$ variation at low $T$ provides an activation energy close to $E_{\eta}$. However, no attempt is made to fit that part of the plot; from the discussion in Sect. 2, it is clear that using $R_{\mathrm{b}}$ for the Ps radius in Eq. (5) (C2) leads to calculated $k^{\prime}$ values that are too low by about a factor of 2 . 
(ii) After the maximum in $k^{\prime}$ is reached, the chemically activated (activation energy: $\left.E^{*}\right)$ process would become important, and the authors propose that $k^{\prime}$ is then proportional to $\eta \exp \left(-E^{*} /\left(k_{\mathrm{B}} T\right)\right)$ : provided that $E_{\eta}>E^{*}$, this would result in an anti-Arrhenius behaviour with a (negative) activation energy: $E_{3}=E_{\eta}-E^{*}$. To assess the plausibility of the above hypothesis, a quantitative approach to the data in Fig. 2 is described in the following.

For a reaction involving two steps, kinetic theories express the overall rate constant as

$$
k^{\prime}=k_{1} k^{*} /\left(k_{1}+k^{*}\right) .
$$

Constant $k_{1}$ is given by Eq. (10) and for the activated rate constant one would have, following the authors' suggestion:

$$
k^{*}=D \exp \left(E_{3} /\left(k_{\mathrm{B}} T\right)\right) .
$$

Equations (12) and (13) lead to calculated $k^{\prime}$ values which are very similar to those shown in Fig. 2. The fitting parameters for $k_{1}$ are the same as obtained from the conventional approach: $A=1.46 \mathrm{M}^{-1} \mathrm{~ns}^{-1} \mathrm{~K}^{-1}$ and $E_{1}=0.068 \mathrm{eV}$. However, using $R_{\mathrm{b}}=4.1 \mathrm{~nm}[2]$ as the Ps radius yields $R_{\mathrm{S}}=3.5 \mathrm{~nm}$, a quite unrealistic value. For $k^{*}$, the parameters are $D=9.39 \times 10^{3} \mathrm{~s}^{-1}$ and $E_{3}=0.512 \mathrm{eV}$. The latter is almost as high as $E_{2}$ from Eq. (11) and much higher than $E_{\eta}$ : this is contrary to the initial hypothesis on $E_{3}$ and thus shows internal inconsistency. In fact, the steepness of the decrease in $k^{\prime}$ at high $T$ cannot be accounted for with an activation energy that would be smaller than $E_{\eta}$.

\section{Conclusions}

This survey shows that many important unknowns still exist regarding Ps dynamics. Most generally, it appears that two concepts that are firmly defended on theoretical grounds and should make Ps a peculiar chemical species, namely, time dependence of the reaction rate coefficient (TD) and existence of a bubble state (BS), are not apt to describe the Ps behaviour, whereas the conventional approach leads, in most cases, to satisfactory results. The evidence is such that even in the case of large solutes (micelles), the use of TD and BS remains questionable, in spite of the good quality of the results produced.

Similarly, the use of BS (not to speak of TD) in the particular case of Ps bound-state formation appears to lead to internal inconsistency when quantitatively applied to the kinetics of Ps, even though it may have provided satisfactory results (at least to the authors' judgement) in terms of reaction mechanism. Again, the conventional approach provides results that are much more self-consistent.

The reasons for the success of the conventional calculations are not clear. The hints given in terms of molecular aggregation must be considered with great caution and may be just an ad hoc explanation. For instance, the appearance of such ratios $\rho$ (experimental to calculated reaction rate constants) such as 1, 2, and 4 might well denote some (hopefully, simple) theoretical background still to discover. Several aspects of the problem, such as tunnelling, size of bubble for 
small particles or bubble formation time have not been dealt with in this review, but it is easily shown that their importance is only marginal or can be made so when considering the whole wealth of data available.

The importance of a better understanding of Ps dynamics goes far beyond the fundamental field of Ps chemistry. Deriving reliable quantitative information on chemical systems obviously demands a much better knowledge of the probe than now available. In this quest, specific studies on both experimental and, more importantly, theoretical grounds should be developed.

\section{References}

[1] A.P. Buchikhin, V.I. Goldanskii, V.P. Shantarovich, JETP Lett. 13, 444 (1971).

[2] W.F. Magalhaes, J.C. Abbé, G. Duplâtre, Struct. Chem. 2, 399 (1991).

[3] T. Mukherjee, S.K. Das, B.N. Ganguly, B. Dutta-Roy, Phys. Rev. B 57, 13363 (1998).

[4] S.A. Rice, in: Comprehensive Chemical Kinetics, Eds. C.H. Bamford, C.F.H. Tipper, R.G. Compton, Elsevier, Amsterdam 1985.

[5] F. Bockstahl, G. Duplâtre, Phys. Chem. Chem. Phys. 1, 2767 (1999).

[6] S.J. Tao, J. Chem. Phys. 52, 752 (1970).

[7] G. Duplâtre, in: Positron, Positronium Chemistry, Eds. Y.C. Jean, P.E. Mallon, D.M. Schrader, World Scientific, London 2003, p. 73.

[8] A.L. Fantola-Lazzarini, E. Lazzarini, Chem. Phys. Lett. 113, 380 (1985).

[9] W.J. Madia, A.L. Nichols, H.J. Ache, J. Am. Chem. Soc. 97, 5041 (1975).

[10] J.Ch. Abbé, G. Duplâtre, A.G. Maddock, J. Talamoni, A. Haessler, J. Inorg. Nucl. Chem. 11, 2603 (1981).

[11] G. Duplâtre, J. Talamoni, J.Ch. Abb, A. Haessler, Radiat. Phys. Chem. 5, 531 (1984).

[12] S.J. Tao, T.M. Kelly, S.Y. Chuang, J.M. Wilkenfeld, Appl. Phys. 3, 31 (1974).

[13] F. Didierjean, G. Duplâtre, J. Ch. Abb, Radiat. Phys. Chem. 38, 413 (1991).

[14] F. Bockstahl, I. Billard, G. Duplâtre, A. Bonnenfant, Chem. Phys. 236, 393 (1998).

[15] T. Messaoud, G. Duplâtre, G. Waton, B. Michels, Phys. Chem. Chem. Phys. 7, 3839 (2005).

[16] G. Duplâtre, in: Positronium and Positronium Chemistry, Ed. Y.C. Jean, World Scientific, London 1990, p. 329.

[17] Y. Kobayashi, Y. Ujihira, J. Phys. Chem. 65, 2455 (1981).

[18] Y. Kobayashi, J. Chem. Soc. Faraday Trans. 87, 3641 (1991).

[19] D. Gangopadhyay, B. N. Ganguly, T. Mukherjee, B. Dutta-Roy, J. Chem. Phys. 111, 9867 (1999) and Chem. Phys. Lett. 318, 161 (2000). 